\title{
Checklist of the benthic marine macroalgae from Algeria, part II: Ulvophyceae
}

\author{
Nora OULD-AHMED ${ }^{1, *}$, Amelia GÓMEZ GARRETA ${ }^{2} \&$ María Antonia RIBERA SIGUAN $^{3}$ \\ ${ }^{1}$ Ecole Nationale Supérieure des Sciences de la Mer et de l'Aménagement du Littoral, Campus Universitaire de Dely-Îbrahim, \\ B.P. 19, Bois des cars, 16320 Alger, Algeria. \\ ${ }^{2,3}$ Laboratori de Botànica, Facultat de Farmàcia i Ciències de l'Alimentació, Universitat de Barcelona, \\ Av. Joan XXIII s/n, 08028 Barcelona, Spain. \\ ${ }^{*}$ Corresponding author: noraouldahmed@yahoo.fr, https://orcid.org/0000-0002-1250-6004 \\ 2ameliagomez@ub.edu, https://orcid.org/0000-0002-9859-2782 \\ ${ }^{3}$ riberasiguan@ub.edu, https://orcid.org/0000-0002-8514-4307
}

\begin{abstract}
The seaweed diversity of the Mediterranean is still not completely known, especially in some areas of its African coasts. As an effort to complete a more detailed catalogue to fill such gap, an updated checklist of seaweeds from Algeria, based on updated literature records, is developed using as starting point the checklist of Perret-Boudouresque and Seridi published in 1989. In the present work, in which we include the Ulvophyceae Mattox \& K.D.Stewart, we list 73 accepted taxa from this group, among which there are 8 new records for Algeria.
\end{abstract}

Keywords. Algerian coast, checklist, green algae, seaweeds, Ulvophyceae.
Resumen. El conocimiento de la diversidad de las algas marinas del Mediterráneo presenta todavía ciertas lagunas, especialmente en algunas áreas de la costa africana. Para completar su catálogo, se está llevando a cabo una revisión crítica y una puesta al día de la flora de algas marinas de Argelia mediante la recopilación y actualización de todas las citas publicadas, tomando como punto de partida la de Perret-Boudouresque y Seridi publicada en 1989. En el presente trabajo, en el que tratamos las Ulvophyceae Mattox \& K.D.Stewart, catalogamos un total de 73 táxones aceptados de este grupo, entre los que se encuentran 8 nuevas citas para Argelia.

Palabras clave. Algas marinas, algas verdes, catálogo, costa argelina, Ulvophyceae.

How to cite this article: Ould-Ahmed N., Gómez Garreta A. \& Ribera Siguan M.A. 2019. Checklist of the benthic marine macroalgae from Algeria, part II: Ulvophyceae. Anales del Jardín Botánico de Madrid 76 (2): e087. https://doi.org/10.3989/ajbm.2471

Title in Spanish: Catálogo de las macroalgas bénticas marinas de Argelia, parte II: Ulvophyceae.

Received: 27-V-2019; accepted: 23-IX-2019; published on-line: 20-XI-2019; Associate Editor: A. Flores.

\section{INTRODUCTION}

The seaweeds of the Algerian coast have been well studied by classical French phycologists (Montagne 1838, 1846-1849, 1856; Debray 1893, 1897; and several contributions carried out by J. Feldman and G. FeldmanMazoyer between 1931 and 1961, listed in PerretBoudouresque \& Seridi 1989). Perret-Boudouresque \& Seridi (1989) published a checklist of the benthic marine macroalgae which compiled the information on this area up to that moment. In 1993, a taxonomical and nomenclatural critical revision of all the Mediterranean green seaweeds (Ulvophyceae Mattox \& K.D.Stewart) was carried out (Gallardo \& al. 1993), and concerning the Algerian flora, the article by Perret-Boudouresque \& Seridi (1989) was used as a reference. In the two last decades, some authors have continued working on the marine flora of Algeria (Ould-Ahmed \& al. 1995; Ould-Ahmed \& Meinesz 1998, 2007; Ould-Ahmed 2005; Seridi \& al. 2007). OuldAhmed \& al. (2013) published the first part of the checklist of Algeria corresponding to the Phaeophyceae Kjellm. In addition, there is more information which is often difficult to consult, since it is mostly included in unpublished papers, such as PhD theses (Seridi 1990, 2007; KadariMéziane 1994; Ould-Ahmed 1994, 2015). Báez \& al. (2004, 2005a, 2005b) used the data of the Algerian species of Cladophora Kütz. compiled in Gallardo \& al. (1993) to carry out a geographical analysis of species richness in the Mediterranean Sea, without new data. The aim of this work is to update the knowledge of the green seaweeds inhabiting the Algeria shores.

\section{MATERIAL AND METHODS}

The list has been compiled following the scheme used in the first part of this series (Ould-Ahmed \& al. 2013). All green algal taxa reported in the literature from Algeria have been searched, critically revised and treated according to current rules of taxonomy and nomenclature in order to compile an updated list of the Algerian Ulvophyceae. 
Taxonomy and nomenclature follow Guiry \& Guiry (2019) and, in some cases, Cormaci \& al. (2014). In the catalogue, orders, genera and species were arranged alphabetically. When a taxon has been recorded under a synonym, this name is cited after the reference. For information included in unpublished works, locations are indicated after the authors' citations (Fig. 1). For each taxon the phytogeographic group is annotated according to Cormaci \& al. (1982), Giaccone \& al (1985), Furnari \& al. (2003) and Guiry \& Guiry (2019): A = Atlantic; $\mathrm{Ab}=$ Boreo-Atlantic; $\mathrm{Abt}=$ Boreo-tropical Atlantic; $\mathrm{AP}=$ Atlanto-Pacific; $\mathrm{At}=$ Atlantic tropical; $\mathrm{C}=$ Cosmopolitan; $\mathrm{CB}=$ Circumboreal; $\mathrm{IA}=$ Indo-Atlantic; IP = Indo-Pacific; $\mathrm{M}=$ Mediterranean; $\mathrm{P}=$ Pantropical; SC $=$ Sub-cosmopolitan. For several species, comments on taxonomic or misidentifications are included. A list of excluded taxa is also given.

\section{RESULTS}

\section{Bryopsidales J.H.Schaffn.}

\section{Bryopsis corymbosa J.Agardh (AP)}

Perret-Boudouresque \& Seridi (1989).

\section{Bryopsis cupressina J.V.Lamour. (M)}

Perret-Boudouresque \& Seridi (1989) as Bryopsis cupressoides J.Agardh var. cupressoides - Kadari-Méziane (1994) as Bryopsis cupressoides var. cupressoides: Tipaza, Bou-Ismail.

Bryopsis cupressina var. adriatica (J.Agardh) M.J.Wynne

Perret-Boudouresque \& Seridi (1989) as Bryopsis cupressoides var adriatica J.Agardh - Kadari-Méziane (1994) as Bryopsis cupressoides var. adriatica: Tipaza, Bou-Ismail.

\section{Bryopsis duplex De Not. (A)}

Perret-Boudouresque \& Seridi (1989) - Kadari-Méziane (1994): Tipaza, Bou-Ismail.

\section{Bryopsis hypnoides J.V.Lamour. (C)}

Perret-Boudouresque \& Seridi (1989) as Bryopsis monoica Funk - OuldAhmed (2015): Tipaza, Ain Tagourait; Alger, Ain Benian.

\section{Bryopsis muscosa J.V.Lamour. (P)}

Perret-Boudouresque \& Seridi (1989) - Seridi (1990): Bourmerdès, Boudouaou; Bouharoun - Ould-Ahmed (1994): Oran, Mers El Hadjaj. Seridi \& al. (2007) - Ould-Ahmed (2015): Oran, Iles Habibas; Arzew.

\section{Bryopsis plumosa (Huds.) C.Agardh (SC)}

Perret-Boudouresque \& Seridi (1989) - Kadari-Méziane (1994): Tipaza, Bou-Ismail - Ould-Ahmed (1994): Oran, Mers El Hadjaj - Ould-Ahmed (2015): Oran, Iles Habibas; Arzew; Tipaza: Chenoua, Anse de Kouali; Alger, Ain Benian.

\section{Bryopsis secunda J.Agardh (M)}

Ould-Ahmed (1994): Oran, Mers El Hadjadj - Ould-Ahmed (2015): Tipaza, Arzew.

\section{Caulerpa cylindracea Sond. (IP)}

Ould-Ahmed \& Meinsez (2007) as Caulerpa racemosa var. cylindracea (Sond.) Verlaque \& al. - Ould-Ahmed (2015) as Caulerpa racemosa var. cylindracea: Tipaza, Bou-Ismail; Alger, Sidi-Fredj, Ain Benian.

\section{Caulerpa prolifera (Forssk.) J.V.Lamour. (P)}

Perret-Boudouresque \& Seridi (1989) - Ould-Ahmed (1994): Oran, Mers El Hadjadj - Ould-Ahmed (2015): Oran, Arzew.

\section{Codium adhaerens C.Agardh (AP)}

Perret-Boudouresque \& Seridi (1989).

According to Gallardo \& al. (1993), the Mediterranean records of this species should be probably referred to Codium effusum. The status of

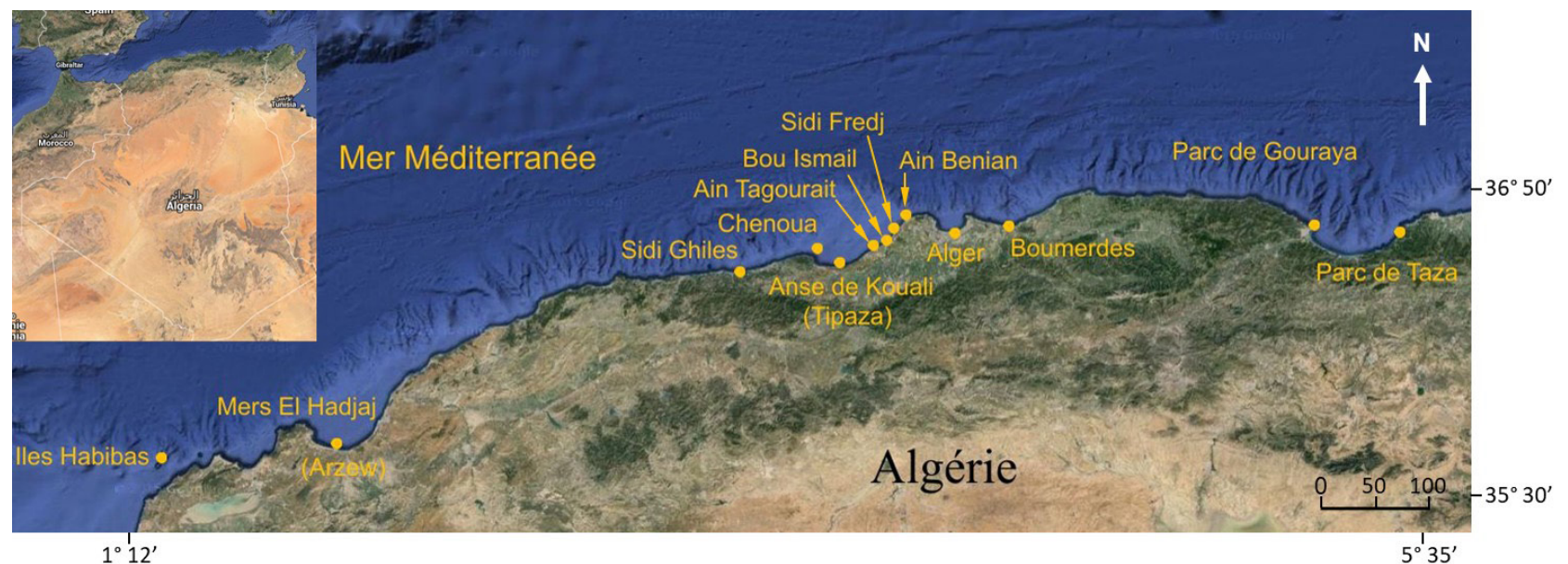

Fig. 1. Geographical distribution of the studied locations along the Algerian coast. 
this species in the Mediterranean and elsewhere outside the north-eastern Atlantic needs reassessment (Guiry \& Guiry 2016).

\section{Codium bursa (Olivi) C.Agardh (Abt)}

Perret-Boudouresque \& Seridi (1989) - Seridi (1990): Surcouf - OuldAhmed (1994): Oran, Mers El Hadjadj - Ould-Ahmed (2015), Oran, Iles Habibas, Arzew; Tipaza, Tipaza, Bou-Ismail; Alger, Ain-Benian; Bejaia, Gouraya; Jijel, Taza.

\section{Codium decorticatum (J.B.Woodw.) M.Howe (IA)}

Perret-Boudouresque \& Seridi (1989) - Ould-Ahmed (1994): Oran, Mers El Hadjaj - Ould-Ahmed (2015): Oran, Arzew, AinTagourait; Bejaia, Gouraya.

\section{Codium effusum (Raf.) Delle Chiaje (IP)}

Perret-Boudouresque \& Seridi (1989) - Seridi (1990): Alger, Bordj El Kiffan, Surcouf.

\section{Codium fragile (Suringar) Har. (AP)}

Seridi (1990): Tamentfoust, Surcouf, Bourmerdès; Boudouaou - OuldAhmed (1994): Oran, Mers El Hadjaj - Seridi \& al. (2007) - Ould-Ahmed (2015): Oran, Arzew; Tipaza, Bou-Ismail; Alger, Sidi-Fredj, Ain Benian; Boumerdès, Boudouaou.

\section{Codium tomentosum Stackh. (AP)}

Perret-Boudouresque \& Seridi (1989) - Ould-Ahmed (1994): Oran, Mers El Hadjadj - Ould-Ahmed (2015): Oran, Arzew; Alger, Ain Benian.

According to Gallardo \& al. (1993), many Mediterranean records of Codium tomentosum should be probably referred to Codium vermilara.

\section{Codium vermilara (Olivi) Delle Chiaje (At)}

Perret-Boudouresque \& Seridi (1989) - Seridi (1990): Alger, Bordj El Kiffan, Tamentfoust, El Marsa, Surcouf; Bourmerdès, Boudouaou Seridi \& al. (2007).

\section{Derbesia tenuissima (Moris \& De Not.) P.Crouan \& H.Crouan (SC)}

Perret-Boudouresque \& Seridi (1989) - Seridi (1990): Alger, Sidi-Fredj Ould-Ahmed (2015): Oran, Arzew; Tipaza, Chenoua, Ain Tagourait Kadari-Méziane (1994) as Halicystis parvula F.Schmitz ex Murray: Tipaza, Bou-Ismail - Ould-Ahmed (1994) as Halicystis parvula: Oran, Mers El Hadjadj.

\section{Flabellia petiolata (Turra) Nizam. (At)}

Perret-Boudouresque \& Seridi (1989) as Udotea petiolata (Turra) Børgesen - Seridi (1990) as Udotea petiolata (Turra): Alger, Bordj El Kiffan, Tamentfoust, Surcouf; Tipaza, Tipaza - Ould-Ahmed (1994) as Udotea petiolata: Oran, Mers El Hadjadj - Ould-Ahmed (2015): Oran, Iles Habibas, Arzew; Tipaza, Anse de Kouali, Bou-Ismail; Alger, Ain Benian; Bejaia, Gouraya; Jijel, Taza.

\section{Halimeda tuna (J.Ellis \& Sol.) J.V.Lamour. (P)}

Perret-Boudouresque \& Seridi (1989) - Ould-Ahmed (1994): Oran, Mers El Hadjadj - Ould-Ahmed (2015): Oran, Arzew.

\section{Ostreobium quekettii Born. \& Flah. (SC)}

Perret-Boudouresque \& Seridi (1989) - Ould-Ahmed (1994): Oran, Mers El Hadjadj - Ould-Ahmed (2015): Oran, Iles Habibas, Arzew.

Pedobesia simplex (Menegh. ex Kütz.) M.J.Wynne \& F.Leliaert (CB)

Perret-Boudouresque \& Seridi (1989) as Pedobesia lamourouxii (J.Agardh) Feldmann \& al.

Pedobesia solieri J.Feldm. ex Abélard \& Knoepffler, Perret-Boudouresque \& Seridi (M)

Perret-Boudouresque \& Seridi (1989).

\section{Penicillus capitatus Lam. (At)}

Perret-Boudouresque \& Seridi (1989) as Espera mediterranea Decne.

\section{Pseudobryopsis myura (J.Agardh) Berthold (M)}

Perret-Boudouresque \& Seridi (1989) as Trichosolen myurus (J.Agardh) W.R.Taylor.

\section{Pseudochlorodesmis furcellata (Zanardini) Børgesen} (SC)

Perret-Boudouresque \& Seridi (1989) - Ould-Ahmed (1994): Oran, Mers El Hadjadj - Ould-Ahmed (2015): Oran, Iles Habibas, Arzew; Bejaia, Gouraya; Jijel, Taza.

\section{Cladophorales Haeckel}

\section{Acrocladus pellucidus (Huds.) Boedeker (IA)}

Perret-Boudouresque \& Seridi (1989) as Cladophora pellucida (Huds.) Kütz.

\section{Anadyomene stellata (Wulfen) C.Agardh (P)}

Perret-Boudouresque \& Seridi (1989).

\section{Chaetomorpha aerea (Dillwyn) Kütz. (C)}

Perret-Boudouresque \& Seridi (1989) - Seridi (1990): Alger, Bordj El Kiffan, Sidi-Fredj; Boumerdès, Boudouaou; Tipaza, Tipaza; KadariMéziane (1994): Tipaza, Bou-Ismail - Ould-Ahmed (1994): Oran, Mers El Hadjadj - Seridi \& al. (2007) - Ould-Ahmed (2015): Oran, Iles Habibas, Arzew; Tipaza, Cherchell, Ain Tagourait, Anse de Kouali, BouIsmail; Alger, Sidi-Fredj, Ain Benian; Boumerdès, Boudouaou; Bejaia, Gouraya; Jijel, Taza.

According to Cormaci \& al. (2014) this species is conspecific with Chaetomorpha linum.

\section{Chaetomorpha ligustica (Kütz.) Kütz. (C)}

Perret-Boudouresque \& Seridi (1989) as Chaetomorpha capillaris (Kütz.) Børgesen - Seridi (1990) as Chaetomorpha capillaris: El Marsa, Surcouf, Boumerdès, Boudouaou, Alger, Sidi-Fredj, Tipaza, Tipaza - Kadari-Méziane (1994) as Chaetomorpha capillaris: Tipaza, Bou-Ismail - Ould-Ahmed (1994): Oran, Mers El Hadjaj - Seridi \& al. (2007) as Chaetomorpha mediterranea (Kütz.) Kütz. - Ould-Ahmed (2015) as Chaetomorpha capillaris: Oran, Iles Habibas, Arzew; Tipaza, Cherchell, Anse de Kouali, Bou-Ismai; Jijel, Taza. 


\section{Chaetomorpha linum (O.F.Müll.) Kütz. (C)}

Perret-Boudouresque \& Seridi (1989) as Chaetomorpha linum and as Chaetomorpha chlorotica (Mont.) Kütz. - Ould-Ahmed (2015): Alger, Ain Benian; Boumerdès, Boudouaou.

\section{Cladophora albida (Nees) Kütz. (SC)}

Perret-Boudouresque \& Seridi (1989) - Seridi (1990): Alger, Surcouf, Sidi-Fredj; Tipaza, Bouharoun, Tipaza - Kadari-Méziane (1994): Tipaza, Bou-Ismail - Ould-Ahmed (1994): Oran, Mers El Hadjadj - Ould-Ahmed (2015): Oran, Iles Habibas, Arzew; Alger, Sidi-Fredj, Ain Benian; Bejaia, Gouraya.

\section{Cladophora coelothrix Kütz. (IA)}

Perret-Boudouresque \& Seridi (1989) - Kadari-Méziane (1994): Tipaza, Bou-Ismail - Ould-Ahmed (1994): Oran, Mers El Hadjadj - Ould-Ahmed (2015): Oran, Iles Habibas, Arzew; Alger, Ain Benian.

\section{Cladophora dalmatica Kütz. (IA)}

Perret-Boudouresque \& Seridi (1989) - Kadari-Méziane (1994): Tipaza, Bou-Ismail - Ould-Ahmed (2015): Oran, Iles Habiba; Tipaza, Anse de Kouali.

Cladophora echinus (Biasol.) Kütz. (IA)

Perret-Boudouresque \& Seridi (1989).

\section{Cladophora hutchinsiae (Dillwyn) Kütz. (SC)}

Perret-Boudouresque \& Seridi (1989) - Seridi (1990): Boumerdès, Boudouaou - Kadari-Méziane (1994): Tipaza, Bou-Ismail - Seridi \& al (2007) - Ould-Ahmed (2015): Alger, Ain Benian.

\section{Cladophora laetevirens (Dillwyn) Kütz. (SC)}

Perret-Boudouresque \& Seridi (1989) - Seridi (1990): Alger, Surcouf; Tipaza, Tipaza - Ould-Ahmed (1994): Oran, Mers El Hadjadj - Seridi \& al. (2007) - Ould-Ahmed (2015): Oran, Iles Habibas, Arzew; Tipaza, Cherchell, Chenoua, Ain Tagourait, Anse de Kouali; Alger, Sidi-Fredj, Ain Benian; Jijel, Taza.

\section{Cladophora lehmanniana (Lindenb.) Kütz. (IA)}

Perret-Boudouresque \& Seridi (1989) - Seridi (1990): Alger, Surcouf Ould-Ahmed (2015): Oran, Iles Habibas; Alger, Ain Benian.

\section{Cladophora prolifera (Roth) Kütz. (IA)}

Perret-Boudouresque \& Seridi (1989) - Seridi (1990): Alger, Sidi-Fredj; Tipaza, Tipaza - Ould-Ahmed (1994): Oran, Mers El Hadjadj - Seridi \& al. (2007) - Ould-Ahmed (2015): Oran, Iles Habibas, Arzew; Tipaza, Anse de Kouali; Alger, Sidi-Fredj, Ain Benian; Bejaia, Gouraya,; Jijel, Taza.

Cladophora ruchingeri (C.Agardh) Kütz. (Ab)

Perret-Boudouresque \& Seridi (1989)

\section{Cladophora rupestris (L.) Kütz. (AP)}

Perret-Boudouresque \& Seridi (1989) - Seridi (1990): Alger, Bordj El Kiffan, Surcouf - Kadari-Méziane (1994): Tipaza, Bou-Ismail - OuldAhmed (1994): Oran, Mers El Hadjadj - Seridi \& al. (2007) - OuldAhmed (2015): Oran, Iles Habibas, Arzew; Tipaza, Anse de Kouali; Alger, Sidi-Fredj, Ain Benian; Boumerdès, Boudouaou; Bejaia, Gouraya.

\section{Cladophora sericea (Huds.) Kütz. (SC)}

Perret-Boudouresque \& Seridi (1989) - Kadari-Méziane (1994): Tipaza, Bou-Ismail - Ould-Ahmed (2015): Oran, Iles Habibas; Alger, Ain Benian.

Cladophora vagabunda (L.) Hoek (SC)

Perret-Boudouresque \& Seridi (1989).

Cladophoropsis membranacea (Bang ex C.Agardh) Børgesen (SC)

Perret-Boudouresque \& Seridi (1989).

Microdictyon umbilicatum (Velley) Zanardini (IP)

Ould-Ahmed (2015) as Microdictyon tenuius J.E.Gray: Oran, Iles Habibas.

Rhizoclonium riparium (Roth) F.L.Harv. sensu Leliaert \& Boedekeer (Abt)

Perret-Boudouresque \& Seridi (1989) - Ould-Ahmed (1994): Oran, Mers El Hadjadj - Ould-Ahmed (2015): Oran, Iles Habibas, Arzew.

Valonia macrophysa Kütz. (P)

Perret-Boudouresque \& Seridi (1989) - Ould-Ahmed (1994): Oran, Mers El Hadjadj - Ould-Ahmed (2015): Oran, Arzew, Chenoua; Alger, Ain Benian.

\section{Valonia utricularis (Roth) C.Agardh $(\mathrm{P})$}

Perret-Boudouresque \& Seridi (1989) - Seridi (1990): Alger, Bordj El Kiffan, Surcouf; Tipaza, Bouharoun - Ould-Ahmed (1994): Oran, Mers El Hadjadj - Ould-Ahmed (2015): Oran, Iles Habibas, Arzew; Tipaza, Cherchell, Chenoua; Alger, Sidi-Fredj; Bejaia, Gouraya.

\section{Dasycladales Pascher}

\section{Acetabularia acetabulum (L.) P.C.Silva (IA)}

Perret-Boudouresque \& Seridi (1989) - Ould-Ahmed (1994): Oran, Mers El Hadjadj - Ould-Ahmed (2015): Oran, Iles Habibas, Arzew; Tipaza, Anse de Kouali; Alger, Sidi-Fredj, Ain Benian; Bejaia, Gouraya.

\section{Dasycladus vermicularis (Scop.) Krasser (At)}

Perret-Boudouresque \& Seridi (1989) - Ould-Ahmed (2015): Tipaza, Anse de Kouali.

Parvocaulis parvulus (Solms) S.Berger, Fettweiss, Gleissberg, Liddle, U.Richter, Sawitzky \& Zuccarello (IP)

Perret-Boudouresque \& Seridi (1989) as Polyphysa parvula (Solms) Schnetter \& Bula Meyer.

\section{Ulotrichales Borzì}

Gomontia polyrhiza (Lagerh.) Born. \& Flah. (SC)

Perret-Boudouresque \& Seridi (1989).

Ulothrix flacca (Dillwyn) Thur. (SC)

Perret-Boudouresque \& Seridi (1989). 


\section{Ulothrix subflaccida Wille (IP)}

Perret-Boudouresque \& Seridi (1989).

\section{Ulvales A.L.Blackman \& Tansley}

Blidingia marginata (J.Agardh) P.J.L.Dang. ex Bliding (SC)

Perret-Boudouresque \& Seridi (1989) - Ould-Ahmed (1994): Oran, Mers El Hadjadj - Ould-Ahmed (2015): Oran, Arzew; Tipaza, Anse de Kouali; Alger, Sidi-Fredj, Ain Benian.

\section{Blidingia minima (Nägeli ex Kütz.) Kylin (SC)}

Ould-Ahmed (1994) as Enteromorpha compressa var. minima (Nägel ex Hauck) Hamel: Oran, Mers El Hadjadj - Ould-Ahmed (2015): Oran, Arzew; Alger, Sidi-Fredj; Boumerdès, Boudouaou.

\section{Entocladia major (J.Feldm.) R.Nielsen (M)}

Perret-Boudouresque \& Seridi (1989) as Acrochaete major (J.Feldm.) M.Perret-Boudouresque \& H.Seridi.

\section{Entocladia flustrae Reinke (Ab)}

Perret-Boudouresque \& Seridi (1989).

Phaeophila dendroides (P.Crouan \& H.Crouan) Batters. (SC)

Perret-Boudouresque \& Seridi (1989) - Ould-Ahmed (2015): Tipaza, Anse de Kouali.

\section{Tellamia contorta Batters (Ab)}

Perret-Boudouresque \& Seridi (1989).

\section{Ulva clathrata (Roth) C.Agardh (C)}

Perret-Boudouresque \& Seridi (1989) as Enteromorpha clathrata (Roth) Grev. and as Enteromorpha ramulosa (Hook.) Smith - OuldAhmed (1994) as Enteromorpha clathrata: Oran, Mers El Hadjadj; and as Enteromorpha ramulosa - Ould-Ahmed (2015): Oran, Iles Habibas, Arzew; Tipaza, Anse de Kouali.

\section{Ulva compressa L. (C)}

Perret-Boudouresque \& Seridi (1989) as Enteromorpha compressa (L.) Nees - Seridi (1990) as Enteromorpha compressa: Alger, Bordj El Kiffan, Surcouf; Bourmerdès, Boudouaou; Tipaza, Bouharoun - Kadari-Méziane (1994) as Enteromorpha compressa: Tipaza, Bou-Ismail - Ould-Ahmed (1994) as Enteromorpha compressa: Oran, Mers El Hadjadj - Seridi \& al. (2007) as Enteromorpha compressa - Ould-Ahmed (2015): Oran, Iles Habibas, Arzew; Tipaza, Chenoua, Anse de Kouali; Alger, Sidi-Fredj, Ain Benian; Boumerdès, Boudouaou; Jijel, Taza.

\section{Ulva fasciata Delile (P)}

Perret-Boudouresque \& Seridi (1989).

\section{Ulva flexuosa Wulfen (C)}

Perret-Boudouresque \& Seridi (1989) as Enteromorpha flexuosa (Wulfen) J.Agardh - Ould-Ahmed (1994) as Enteromorpha flexuosa: Oran, Mers El Hadjadj - Ould-Ahmed (2015): Oran, Arzew; Alger, Ain Benian.

\section{Ulva intestinalis L. (C)}

Perret-Boudouresque \& Seridi (1989) as Enteromorpha intestinalis (L.) Nees - Seridi (1990) as Enteromorpha intestinalis: Alger, Bordj El Kiffan, Surcouf; Tipaza, Bouharoun - Ould-Ahmed (1994) as Enteromorpha intestinalis: Oran, Mers El Hadjadj - Seridi \& al. (2007) as Enteromorpha intestinalis - Ould-Ahmed (2015): Oran, Iles Habibas, Arzew; Tipaza, Cherchell, Chenoua, Ain Tagourait, Anse de Kouali, Bou-Ismail; Alger, Sidi-Fredj, Ain Benian; Boumerdès, Boudouaou; Bejaia, Gouraya.

\section{Ulva linza L. (C)}

Perret-Boudouresque \& Seridi (1989) as Enteromorpha linza (L.) J.Agardh - Seridi (1990) as Enteromorpha linza: Alger, Bordj El Kiffan, Surcouf; Bourmerdès, Boudouaou - Ould-Ahmed (2015): Tipaza, Cherchell, Ain Tagourait, Anse de Kouali; Alger, Sidi-Fredj, Ain Benian.

\section{Ulva prolifera O.F.Müll. (SC)}

Kadari-Méziane (1994) as Enteromorpha prolifera (O.F. Müll.) J.Agardh: Tipaza, Bou-Ismail - Ould-Ahmed (2015): Tipaza, Chenoua.

\section{Ulva rigida C.Agardh (C)}

Perret-Boudouresque \& Seridi (1989) - Seridi (1990): Alger, Bordj El Kiffan, El Marsa, Surcouf; Bourmerdès, Boudouaou; Tipaza, Bouharoun, Tipaza - Kadari-Méziane (1994): Tipaza, Bou-Ismail - Ould-Ahmed (1994): Oran, Mers El Hadjadj - Seridi \& al. (2007) - Ould-Ahmed (2015): Oran, Iles Habibas, Arzew; Tipaza, Cherchell, Ain Tagourait, Anse de Kouali, Bou-Ismail; Alger, Sidi-Fredj , Ain Benian; Boumerdès, Boudouaou.

\section{Ulvella lens P.Crouan \& H.Crouan (SC)}

Ould-Ahmed (2015): Tipaza, Anse de Kouali.

Ulvella pennata (J.Feldm.) Cormaci, G. Furnari \& Alongi (M)

Perret-Boudouresque \& Seridi (1989) as Entocladia pennata (J.Feldm.) R.Nielsen.

Ulvella porphyrae (J.Feldm.) R.Nielsen, O’Kelly \& B.Wysor (M)

Perret-Boudouresque \& Seridi (1989) as Pseudodictyon porphyrae J.Feldm.

Ulvella viridis (Reinke) R.Nielsen, O'Kelly \& B.Wysor (C)

Perret-Boudouresque \& Seridi (1989) as Acrochaete viridis (Reinke) R.Nielsen - Ould-Ahmed (2015) as Acrochaete viridis: Tipaza, Anse de Kouali.

\section{Incertae sedis}

\section{Blastophysa rhizopus Reinke (Abt)}

Perret-Boudouresque \& Seridi (1989) as Blastophysa polymorpha Kjellm. emend Printz - Ould-Ahmed (2015): Tipaza, Anse de Kouali.

\section{Taxa excludenda}

Monostroma grevillei (Thur.) Wittr.

Ould-Ahmed (2015): Tipaza, Anse de Kouali. 
In the Mediterranean marine benthic flora, Cormaci \& al. (2014) included this species in taxa excludenda.

\section{Ulva lactuca $\mathrm{L}$.}

Perret-Boudouresque \& Seridi (1989) - Kadari-Méziane (1994): Tipaza, Bou-Ismail - Seridi \& al. (2007) - Ould-Ahmed (2015): Tipaza, Cherchell, Chenoua, Ain Tagourait, Anse de Kouali, Bou-Ismail; Alger, Sidi-Fredj, Ain Benian; Boumerdès, Boudouaou; Bejaia, Gouraya; Jijel, Taza).

In the Mediterranean marine benthic flora, Cormaci \& al. (2014) included this species in taxa excludenda.

\section{DISCUSSION}

The present checklist encompasses 73 accepted green algal taxa registered to date in Algeria, and two taxa are considered as taxa excludenda. The best represented genera have been Cladophora (13 species), Ulva L. (8 species), Bryopsis J.V.Lamour. (8 species) and Codium Stackh. (7 species). The number of Ulvophyceae registered on the Algerian coasts is low in comparison with its total number for the entire Mediterranean Sea (176 taxa) (Cormaci \& al. 2014), or for the Adriatic Sea (134 taxa) (Antolic \& al. 2001). However, it is similar to other Mediterranean countries such as Greece (95 taxa) (Tsiamis \& al. 2014) and Tunisia (71 taxa) (Guiry \& Guiry 2019), and higher than Libya (31 taxa) (Guiry \& Guiry 2019).

The current catalogue added eight new records to the included in Perret-Boudouresque \& Seridi (1989). Codium fragile and Caulerpa cylindracea are species included in the list of introduced species of the Mediterranean Sea (Zenetos \& al. 2010, 2012). The former is a species originate from the northwestern Pacific and at present with a cosmopolitan distribution. Seridi (1990) cited this species for the first time in Algeria, but later has been cited frequently by other authors. Caulerpa cylindracea is a species introduced from western Australia (Silva \& al. 1996) and in expansion in the Mediterranean Sea. Actually, this species is cited in all the Mediterranean area and in the Atlantic, Pacific and Indic Oceans (Guiry \& Guiry 2019). It was cited for the first time in Algeria by Ould-Ahmed \& Meinesz (2007), and later by Ould-Ahmed (2015) in BouIsmail, Sidi-Fredj and Ain Benian. Three new species belong to the order Ulvales: Ulva prolifera, Ulvella lens and Blidingia minima and two to the order Cladophorales: Chaeotomorpha ligustica and Microdictyon umbilicatum. These five species are cited along all the Mediterranean coasts. Regarding the remaining new species, Bryopsis secunda, is only known from the Mediterranean Sea (Guiry \& Guiry 2019). Cormaci \& al. (2014) indicated that is a rare species and according Pignatti (1962) most of the ancient records are misidentifications for Bryopsis hypnoides.

Biogeographically, the Algerian flora of Ulvophyceae is characterized by a high number of sub-cosmopolitan and cosmopolitan taxa (28 taxa, 38\%) followed by Atlantic taxa (11 taxa, 15\%), Mediterranean and Indo-Atlantic (8 taxa each, 11\%), Pantropical (7 taxa, 10\%) and Atlanto-Pacific and Indo-Pacific (5 taxa each, 7\%). The circumboreal taxa are scarcely represented (1 species). This biogeographical composition of the Algerian flora of Ulvophyceae is similar to the observed in Algerian flora of Phaeophyceae by Ould-Ahmed \& al. (2013).

\section{ACKNOWLEDGEMENTS}

The authors thank the support given by Jordi Rull and Samy OuldAhmed. We are grateful to Samuel Pyke for considerably improving the English of the text.

\section{REFERENCES}

Antolic B., Span A., Zuljevic A. \& Vukovic A. 2001. Check list of the benthic marine macroalgae on the eastern Adriatic coast: I. Chlorophyta. Acta Adriatica 42: 43-58.

Báez J.C., Real R., Vargas J.M. \& Flores-Moya A. 2004. A biogeographical analysis of the genera Audouinella (Rhodophyta), Cystoseira (Phaeophyceae) and Cladophora (Chlorophyta) in the western Mediterranean Sea and Adriatic Sea. Phycologia 43: 404-415. https://doi.org/10.2216/10031-8884-43-4-404.1

Báez J.C., Real R., Vargas J.M. \& Flores-Moya A. 2005a. Chorotypes of seaweeds from the western Mediterranean Sea and the Adriatic Sea: An analysis based on the genera Audouinella (Rhodophyta), Cystoseira (Phaeophyceae) and Cladophora (Chlorophyta). Phycological Research 53: 255-265. https://doi.org/10.1111/j.1440-183.2005.00393.x

Báez J.C., Olivero J., Real R., Vargas J.M. \& Flores-Moya A. 2005 b. Analysis of geographical variation in species richness within the genera Audouinella (Rhodophyta), Cystoseira (Phaeophyceae) and Cladophora (Chlorophyta) in the western Mediterranean Sea. Botanica Marina 48: 30-37. https://doi.org/10.1515/bot.2005.012

Cormaci M., Duro A. \& Furnari G. 1982. Considerazioni sugli elementi fitogeografici della flora algale della Sicilia. Naturalista Siciliano Ser. IV 6 (Suppl.): 7-14.

Cormaci M., Furnari G. \& Alongi G. 2014. Flora marina bentonica del Mediterraneo: Chlorophyta. Bollettino dell'Accademia Gioenia di Scienze Naturali di Catania 47: 11-436.

Debray F. 1893. Liste des algues marines et d'eau douce récoltées jusqu'à ce jour in Algérie. Bulletin Scientifique de la France et de la Belgique 25: 1-19. https://doi.org/10.1080/00378941.1860.10839752

Debray F. 1897. Catalogue des algues du Maroc, d'Algérie et de Tunisie. Librairie Adolphe Jourdan Edit, Alger.

Furnari G., Giaccone G., Cormaci M., Alongi G. \& Serio D. 2003. Biodiversità marina delle coste italiane: catalogo del macrofitobenthos. Biologia Marina Mediterranea 10 (1): 1-482.

Gallardo T., Gómez Garreta A., Ribera M.A., Cormaci M., Furnari G., Giaccone G. \& Boudouresque C.F. 1993. Check-list of Mediterranean Seaweeds, II. Chlorophyceae Wille s.1.. Botanica Marina 36: 399-421. https://doi.org/10.1515/botm.1993.36.5.399

Giaccone G., Colonna P., Graziano C., Mannino A.M., Tornatore E., Cormaci M., Furnari G. \& Scammacca B. 1985. Revisione della flora marina di Sicilia e isole minori. Bollettino della Accademia Gioenia di Scienze Naturali di Catania 18: 537-781. https://doi.org/10.5962/bhl.title.11386

Guiry M.D. \& Guiry G.M. 2019. AlgaeBase. World-wide electronic publication. National University of Ireland, Galway. Website: http://www.algaebase.org [accessed 27 Sep. 2016]. 
Kadari-Méziane Y. 1994. Contribution à l'étude de l'impact de la pollution sur la distribution spatio-temporelle des peuplements phytobenthiques dans la baie de Bou-Ismail (Algérie). Ph.D. dissertation, ENS, Alger.

Montagne C. 1838. Cryptogames algériennes, ou plantes cellulaires recueillies par M. Roussel aux environs d'Alger, et publiées par le Docteur Camille Montagne. Annales des Sciences Naturelles, Botanique, série 2, 10: 268-279. https://doi.org/10.1080/037454809495195

Montagne C. 1846-1849. Ordo I. Phyceae Fries. In Bory de Saint-Vincent J.B.G.M. \& Durieu de Maisonneuve M.C., Exploration scientifique de l'Algérie. Botanique. Imprimerie impériale, Paris.

Montagne C. 1856. Sylloge Generum Specierumque Cryptogamarum. J.B. Bailliere Edit., Paris. https://doi.org/10.5962/bhl.title.5403

Ould-Ahmed N. 1994. Étude des espèces phytobenthiques au voisinage de la centrale thermique de Mers El Hadjdj (Golfe d'Arzew; Ouest algérien). Mention particulière sur une espèce remarquable Chlorophyte, Caulerpales: Caulerpa prolifera (Forsskal) Lamouroux. Ph.D. dissertation, ISMAL, Alger.

Ould-Ahmed N. 2005. Note sur les algues des côtes algériennes. INOCCOMSTECH-ISESCO. The Protection of Costal and Environnement: 55-60.

Ould-Ahmed N. 2015. Les algues des côtes algériennes. Connaissance, Caractérisation, Conservation et Utilisation. Ph.D. dissertation, ENSA Alger.

Ould-Ahmed N. \& Meinesz A. 1998. Note sur la prédominance d'une Chlorophyte Caulerpale. Caulerpa prolifera (Forsskal) Lamouroux, au voisinage d'une centrale à production d'électricité (804 M.W.) de Mers-El-Hadjadj (Golfe d'Arzew ; Ouest algérien). Rapport Commission International Mer Méditerranée (Croatie) 35(2): 566-567. https://doi.org/10.1515/botm.1979.22.2.117

Ould-Ahmed N. \& Meinesz A. 2007. First record of the invasive alga Caulerpa racemosa (Caulerpales, Chlorophyta) on the coast of Algeria. Cryptogamie Algologie 28: 303-305. https://doi.org/10.3391/ai.2010.5.s1.020

Ould-Ahmed N., Bounaga N. \& Cirik C. 1995. Le peuplement algal au voisinage de la centrale thermique de Mers-El Hadjadj (Golfe d'Arzew; ouest algérien): Affinités biogéographiques. Rapport Commission International Mer Méditerranée (Malte) 34: 38.

Ould-Ahmed N., Gómez Garreta A., Ribera Siguan M.A. \& Bouguedoura N. 2013. Checklist of the benthic marine macroalgae from Algeria.
I. Phaeophyceae. Anales del Jardin Botánico de Madrid 70: 136-143. https://doi.org/10.3989/ajbm.2349

Perret-Boudouresque M. \& Seridi H. 1989. Inventaire des algues marines benthiques d'Algerie. GIS Posidonie publ., Marseille.

Pignatti S. 1962. Le specie mediterranee del genere Bryopsis (Chlorophyceae, Siphonales). Atti del Reale Istituto Veneto di Scienze, Lettere ed Arti 120: 31-58. https://doi.org/10.2307/476535

Seridi H. 1990. Étude des algues marines benthiques de la région d'Alger. Ph.D. dissertation, USTHB, Alger.

Seridi H. 2007. Étude de la flore algale de l'Algérie. Etude phytosociologique des peuplements algaux photophiles de l'infralittoral supérieur de substrat dur. Ph.D. dissertation, USTHB, Alger.

Seridi H., Ruitton S. \& Boudouresque C.F. 2007. Is it possible to calibrate the pollution level of the region of Algiers (Mediterranean Sea) by exploiting marine macrophytes? Comptes Rendus Biologies 330: 606614. https://doi.org/10.1016/j.crvi.2007.05.001

Silva P.C., Basson P.W. \& Moe R.L. 1996. Catalogue of the benthic marine algae of the Indian Ocean. University of California Publications in Botany 79: 1-1259.

Tsiamis K., Panayotidis P., Economou-Amilli A. \& Katsaros C. 2014. Seaweeds of the Greek coasts. II. Ulvophyceae. Mediterranean Marine Science 15: 449-461. https://doi.org/10.12681/mms.574

Zenetos A., Gofas S., Verlaque M., Cinar M.E., García Raso J.E., Bianchi C.N., Morri C., Azzurro E., Bilecenoglu M., Froglia C., Siokou I., Violanti D., Sfriso A., San Martín G., Giangrande A., Katagan T., Ballesteros E., Ramos-Espla A., Mastrototaro F., Ocaña O., Zingone A., Gambi M.C. \& Streftaris N. 2010. Alien species in the Mediterranean Sea by 2010. A contribution to the application of European Union's Marine Strategy Framework Directive (MSFD). Part I. Spatial distribution. Mediterranean Marine Science 11 (2): 381-493. https://doi.org/10.12681/mms.49

Zenetos A., Gofas S., Morri C., Rosso A., Violanti D., García Raso J.E., Cinar M.E., Almogi-Labin A., Ates A.S., Azzurro E., Ballesteros E., Bianchi C.N., Bilecenoglu M., Gambi M.C., Giangrande A., Gravili C., Hyams-Kaphzan O., Karachle P.K., Katsanevakis S., Lipe L., Mastrototaro F., Mineur F., Pancucci-Papadopoulou M.A., Ramos-Esplá A., Salas C., San Martin G., Sfriso A., Streftaris N. \& Verlaque M. 2012. Alien species in the Mediterranean Sea by 2012. A contribution to the application of European Union's Marine Strategy Framework Directive (MSFD). Mediterranean Marine Science 13 (2): 328-352. https://doi. org $/ 10.12681 / \mathrm{mms} .327$ 